\title{
Kyphoplasty for Vertebral Compression Fracture Via a UnI-PEdicular ApProach
}

\author{
M. Melvin Hu, MD, PhD, Clifford J. Eskey, MD, PhD, Samuel C. Tong, MD, Raul G. Nogueira, MD \\ Stuart R. Pomerantz, MD, James D. Rabinov, MD, Johnny C. Pryor, MD, and Joshua A. Hirsch, MD
}

Background: Percutaneous kyphoplasty using a bone expander polymer device, such as percutaneous vertebroplasty and balloon kyphoplasty, is a therapeutic intervention for painful osteoporotic vertebral body compression fractures. Typically the procedure involves placement of bilateral Sky Bone Expanders in the fractured vertebral body via a transpedicular approach. We describe performance of "SKy" phoplasty using the Disc-O-Tech Sky Bone Expander (DiscO-Tech Medical Technologies, Herzliya, Israel, and Monroe Township, New Jersey) via a unilateral transpedicular approach. The advantage of a unilateral approach is that it reduces the risks associated with large-bore needle placement. These risks include pedi-

Like percutaneious vertebroplasty and balloon kyphoplasty, percutaneous "SKy" phoplasty using the Disc-OTech Sky Bone Expander polymer device (Disc-O-Tech Medical Technologies, Herzliya, Israel, and Monroe Township, New Jersey) is a therapeutic intervention per-

From: MGH Pain Center, Endovascular Neurosurgery and Interventional Neuroradiology, Neurosurgical Service and Departments of Radiology, and Anesthesia and Critical Care, Massachusetts General Hospital, Harvard Medical School, Boston, Massachusetts

Address correspondence:

Joshua A. Hirsch, MD

Director, Endovascular Neurosurgery and Interventional Neuroradiology

Massachusetts General Hospital, Gray 289

55 Fruit Street, Boston, MA 02114

E-mail: jahirsch@partners.org

Disclaimer: There was no external funding in the

preparation of this manuscript.

Conflict of Interest: None

Manuscript received on $7 / 1 / 2005$

Revision submitted on $8 / 19 / 2005$

Accepted for publication on $9 / 1 / 2005$

The Disc-O-Tech SKy Bone Expander products and/ or "Sky" phoplasty technique mentioned in this article are patent pending and trademark pending in the United States. cle fracture, medial transgression of the pedicle or transgression into the spinal canal, nerve injury, cement leakage along the cannula tract, and spinal epidural hematoma. Additionally, using a unilateral approach reduces operative time and costs.

Case Illustration: A 68-year-old man with osteoporosis presented with severe upper back pain which occurred following a fall. The pain was reproducible on palpation of the L1 spinous process. A lumbar spine magnetic resonance imaging (MRI) with STIR (short tau inversion recovery) sequence demonstrated an acute L1 vertebral body compression fracture. A L1 "SKy"phoplasty was performed using a single Sky Bone Expander polymer device via a unilateral transpe- dicular approach. The patient reported immediate relief of pain after the procedure. $\mathrm{He}$ denied any residual back pain at his followup visit. He was able to resume his normal activities including walking, which had been inhibited by pain prior to the procedure.

Conclusion: "SKy"phoplasty can be performed using a single Sky Bone Expander via a unilateral pedicular approach. The key is a medial needle trajectory with a final Sky Bone Expander position in the midline of the vertebral body.

Keywords: Compression fracture, "SKy" phoplasty, osteoporosis, minimally invasive, pain, vertebroplasty

formed for painful osteoporotic vertebral compression fracture (1). The Sky Bone Expander polymer device utilizes a noncompliant plastic polymer tube which radially expands within the collapsed vertebra to reconstruct vertebral height and create a void for subsequent cement or bone filler material. This procedure differs from conventional vertebroplasty and balloon kyphoplasty in that the Sky Bone Expander polymer device is advanced into the fractured vertebral body, the Sky Bone Expander is unsheathed to reduce the fracture and elevate the endplates, and then the Sky Bone Expander is resheathed to produce a cavity into which bone cement, usually polymethylmethacrylate (PMMA) can be injected (1).

The "SKy" phoplasty procedure has consisted of insertion of bilateral Sky Bone Expander either via a bilateral transpedicular approach or a bilateral extra-pedicular approach (usually for thoracic vertebra). We describe performance of a procedure known as "SKy" phoplasty with a unilateral Sky Bone Expander via a unilateral transpedicular approach. A unilateral approach offers the potential advantage of reducing the risk associated with large-bore needle placement. These risks include pedicle fracture, medial transgression of the pedicle or transgression into the spinal canal, nerve injury, cement leakage along the cannula tract, and spinal epidural hematoma. Additionally, operative time and cost are reduced with this technique.

\section{Case Illustration}

A 68-year-old man with osteoporosis presented with severe upper back pain of 1 week which he reported had a clear onset following a fall. The back pain was refractory to conservative treatment including narcotic pain medication and back brace, and rendered him unable to ambulate. He denied radicular symptoms, bowel, or bladder dysfunctions. He was admitted to the hospital for pain control. He had associated diagnoses of osteoporosis, history of alcoholism, cirrhosis, psoriasis, hypertension, and peripheral neuropathy. His medications included Fosamax, MS Contin, Ativan, Lexapro, Ecotrin, amitriptyline. On physical examination, exquisite pain was reproduc- 
ible on palpation of the L1 spinous process. There were no radicular findings and his neurological examination was unremarkable with normal motor strength, reflexes, sensation, and rectal tone.

A spine magnetic resonance imaging (MRI) study was performed which included T1-weighted, T2-weighted, and STIR (short tau inversion recovery) sequences. These demonstrated an acute L1 vertebral compression fracture with associated marrow edema (Fig. 1).

Percutaneous L1 "SKy"phoplasty was performed at the NEUROSTAR biplane angiography unit (Boston, MA) using a Disc-O-Tech Sky Bone Expander polymer device via a unilateral transpedicular approach. Briefly, after conscious sedation and administration of a pre-procedure dose of intravenous cefazolin, the patient was carefully positioned prone on the fluoroscopy table. Using sterile technique, local $1 \%$ lidocaine was administered subcutaneously at the planned needle entry site for the left L1 pedicle which was localized by fluoroscopy. A small skin incision was made and an 11-gauge Jamshidi ${ }^{\oplus}$ type needle was advanced through the left L1 pedi- cle into the L1 vertebral body under intermittent biplane (anteroposterior and lateral) fluoroscopy. Special care was taken to achieve a medial trajectory of the needle and a final midline position of the needle tip in the vertebral body.

Exchange for a trocar was then made over a K-wire. A handheld bone drill was passed through the outer cannula of the trocar over the K-wire and used to create a $4.0 \mathrm{~mm}$ channel through the pedicle and vertebral body up to a few millimeters from the anterior cortex (Fig. 2).

The Sky Bone Expander was then positioned within the vertebral body and expanded under direct fluoroscopy in a controlled manner (Fig. 3). After vertebral endplate height restoration and void creation, the device was slowly reduced to its collapsed configuration and removed. PMMA was prepared with additional barium sulfate. When satisfactory consistency was achieved, PMMA was injected using a commercially available cement delivery system kit under direct fluoroscopic visualization into the cavity in the vertebral body created by the Sky Bone Expander (Fig. 4). A total of $5 \mathrm{~mL}$ of cement was administered which produced an excellent

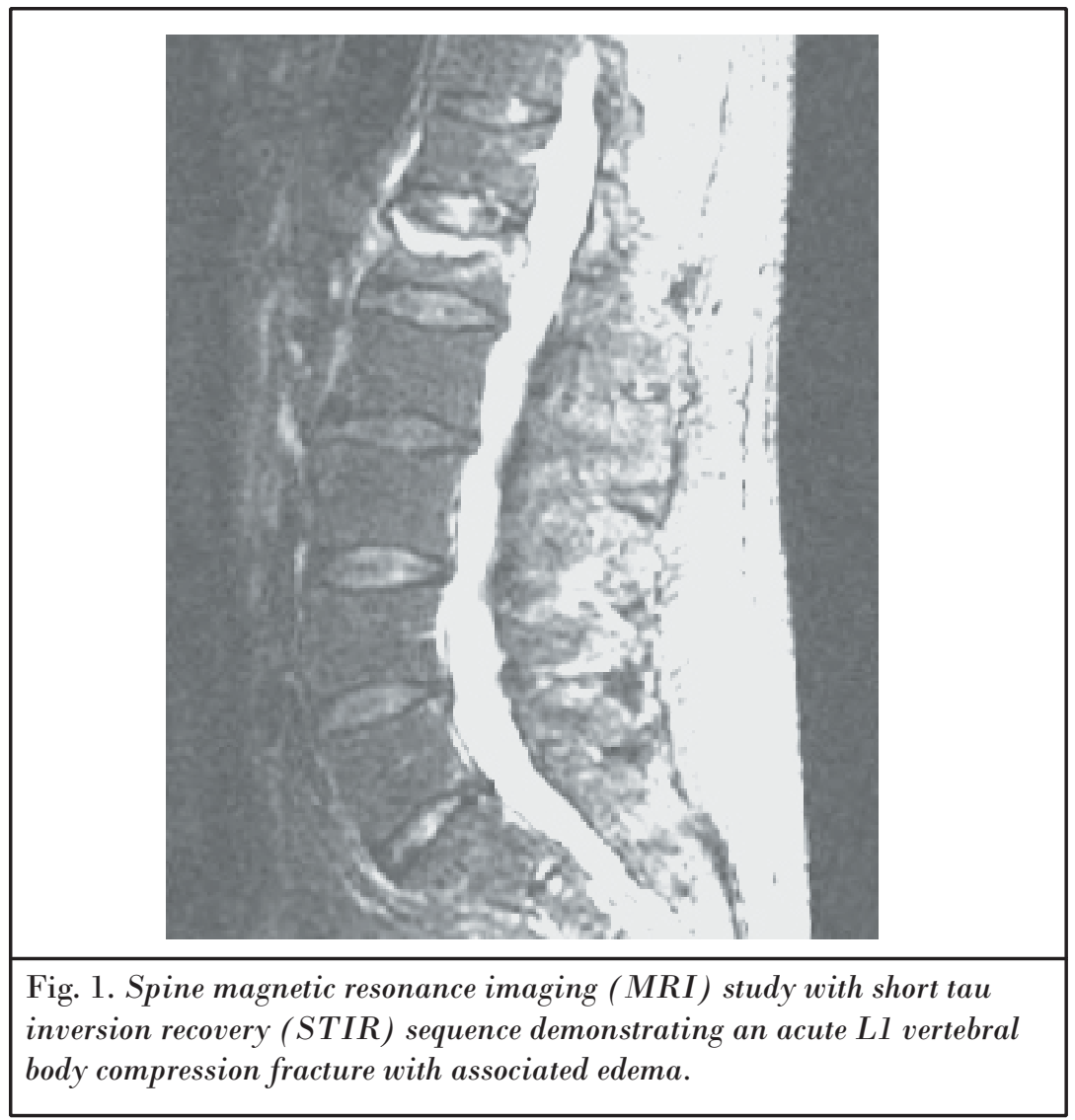

filling of the vertebral body cavity. All instrumentation was removed at the end of the procedure.

The patient reported immediate pain relief. He was able to ambulate without pain after a 2-hour recovery period.

\section{DISCUSSION}

Percutaneous "SKy"phoplasty using the Disc-O-Tech Sky Bone Expander polymer device is a new technique for treatment of painful osteoporotic vertebral body compression fractures (1). The procedure conventionally involves insertion of bilateral Sky Bone Expanders. We describe performance of "SKy" phoplasty using a single unilateral Sky Bone Expander via a unilateral transpedicular approach.

The rationale for the conventional use of bilateral approach is to achieve adequate endplate elevation with two Sky Bone Expanders and to create a large enough cavity for maximal cement filling. With conventional needle trajectories, the Sky Bone Expanders remain ipsilateral, thus necessitating bilateral Sky Bone Expanders to cover the expanse of the vertebral body. In the procedure that we describe, we perform a medially directed trajectory with the final destination of the Sky Bone Expander in the midline of the vertebral body. A midline-positioned Sky Bone Expander can be inflated to create a large enough cavity in the midline of the vertebral body.

The advantage of a unilateral Sky Bone Expander is reducing the risk associated with large-bore needle placement. These risks include pedicle fracture, medial transgression of the pedicle or transgression into the spinal canal, nerve injury, cement leakage along the cannula tract, and spinal epidural hematoma. Though rare, such adverse events have been reported in most of the published case series. Coumans et al (2) described a large series of 188 kyphoplasty procedures. There were only five cases of cement extravasation, and three were along the cannula tract.

Nussbaum et al (3) reviewed complications associated with vertebroplasty and kyphoplasty as reported to the Food and Drug Administration Medical Device-related web site. It was found that kyphoplasty may have an increased risk of pedicle fracture that can lead to spinal compression. At least five of the 20 spinal compressions associated with kyphoplas- 


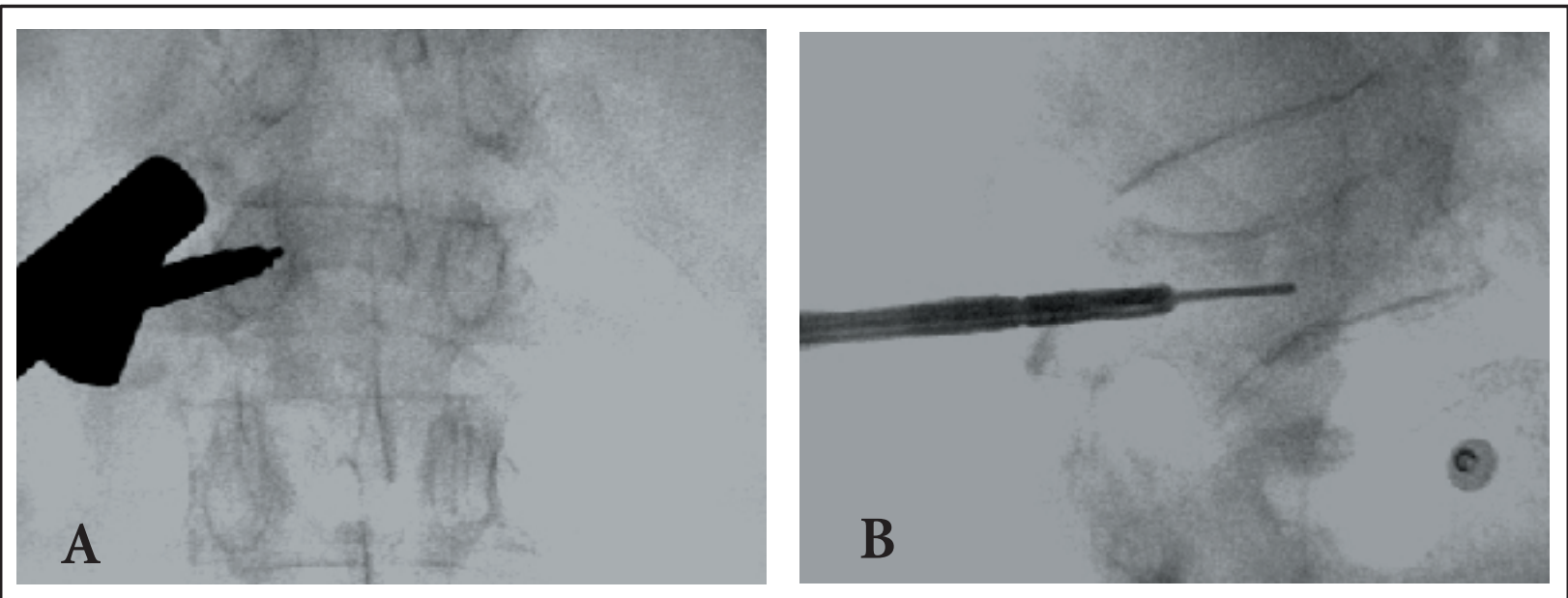

Fig. 2. A) Antero-posterior, and B) lateral fluoroscopic image of medially-directed working cannula and drill trocar.

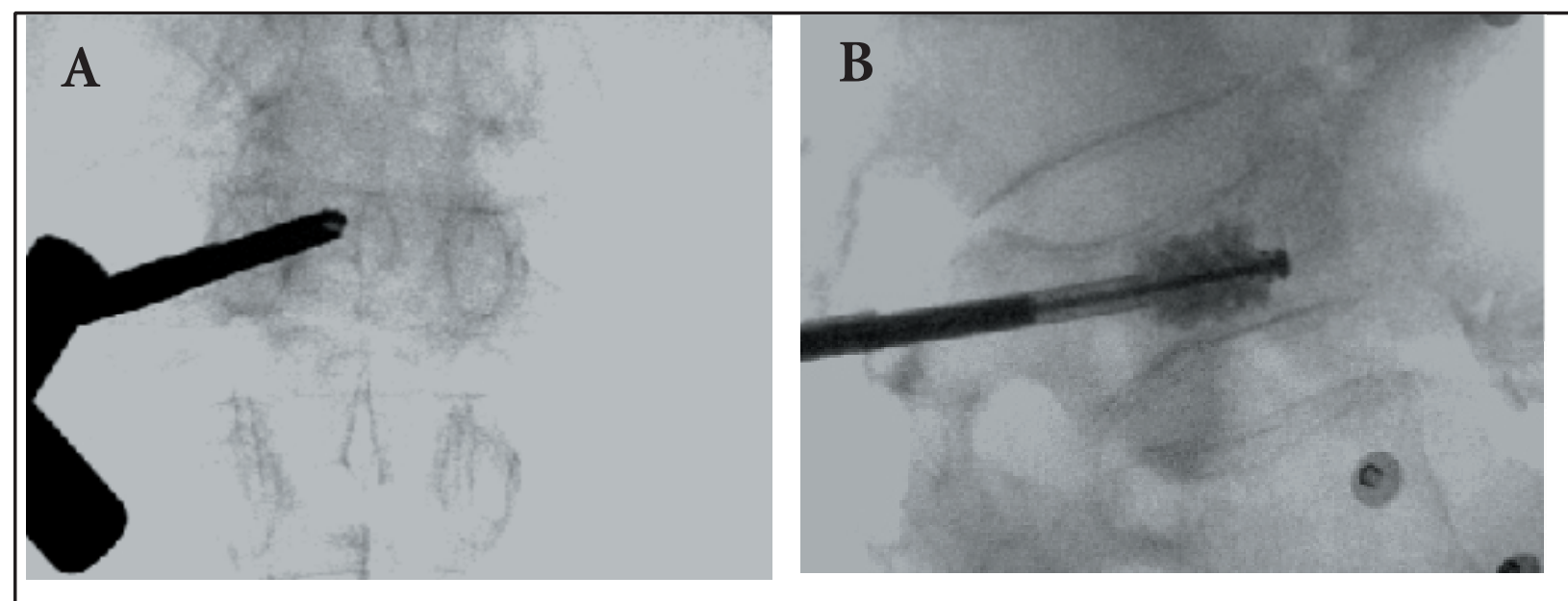

Fig. 3. A) Antero-posterior, and B) lateral fluoroscopic image of Sky Bone Expander in the midline of the fractured vertebral body.
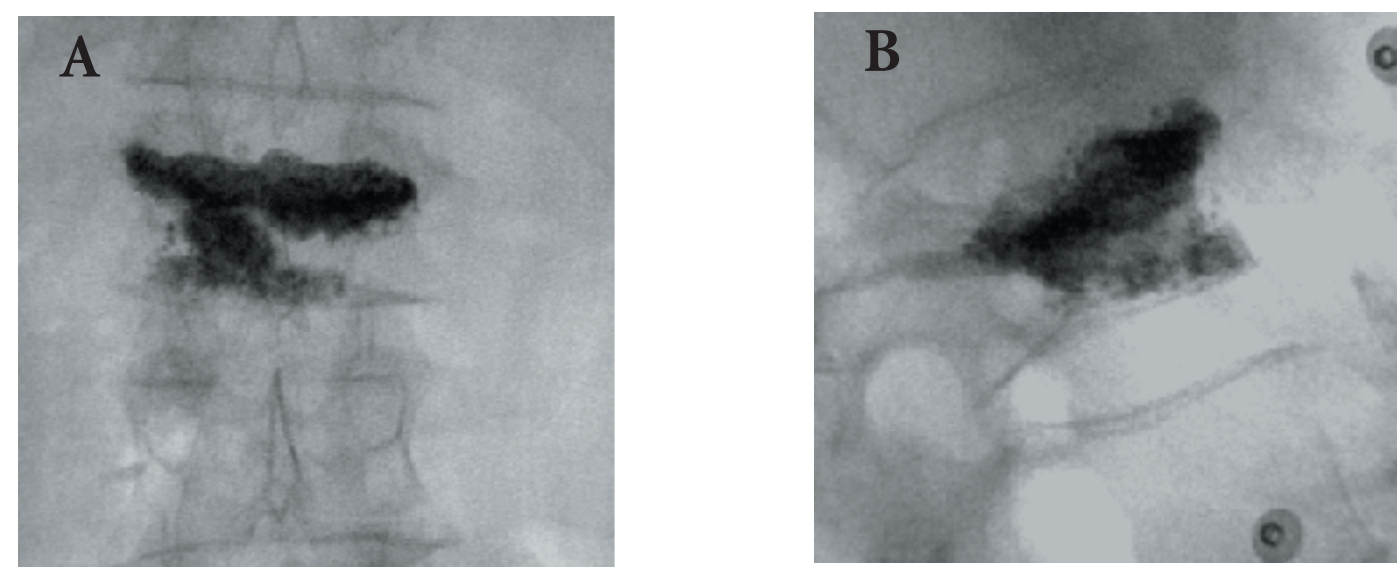

Fig. 4. A) Antero-posterior, and B) lateral fluoroscopic image of polymethylmethacrylate filling the cavity within the fractured vertebral body. 
ty were caused by breakage of the pedicle during insertion of the cannula; of the remaining 15 spinal compressions that developed, only two reports specified that a pedicle fracture was absent on postoperative imaging.

The incidence of such events may be reduced if unilateral rather than bilateral cannulas are placed. Additionally, operative time and cost are reduced. In the procedure that we described, the time required for the procedure was less than 45 minutes. Typically, when we have performed "SKy" phoplasty with bilateral Sky Bone Expanders, the total procedure time is close to one hour.

The procedure was well tolerated by the patient with excellent clinical and radiographic result, which we believe is comparable to "SKy"phoplasty using bilateral Sky Bone Expanders. In a cadaveric study, the biomechanical outcomes including restoration of vertebral body strength, stiffness and height were com- parable for unipedicular versus bipedicular kyphoplasty in experimentally-induced vertebral compression fractures (4). No study has directly compared unilateral versus bilateral approaches for balloon kyphoplasty. However, we have described a unilateral approach for balloon kyphoplasty (5).

In the event that "SKy" phoplasty is attempted using a unilateral Sky Bone Expander and the surgeon is not satisfied with the Sky Bone Expander position or the extent of inflation or cavity created, a second contralateral Sky Bone Expander can be placed using the conventional technique. It is thus our belief that, except in rare circumstances where it is known preoperatively that bilateral Sky Bone Expanders will be needed because of unusual pathologic anatomy, that it is beneficial to attempt a unilateral approach with the backup option of placing a second Sky Bone Expander if unsatisfied with the single Sky Bone Expander. The key to the unilateral approach is the medial trajectory of the needle and the final midline position of the Sky Bone Expander. We have found the drill set provided with the Sky Bone Expander allows excellent medialization of the original pathway, particularly the combination of diamond-point and beveled needles, to be the easiest for us to manipulate in directing the medial trajectory necessary to achieve the midline Sky Bone Expander position.

\section{Conclusion}

We describe performance of percutaneous "SKy" phoplasty for osteoporotic vertebral body compression fracture using a unilateral inflatable Sky Bone Expander via a unilateral transpedicular approach. The key to the approach is a medial trajectory with a final position of the Sky Bone Expander in the midline of the vertebral body.

\section{Author AfFLLATION:}

M. Melvin $\mathrm{Hu}, \mathrm{MD}, \mathrm{PhD}$

Pain Management Fellow

MGH Pain Center

Dept. of Anesthesia and Critical Care

Massachusetts General Hospital

Harvard Medical School

Boston, MA 02114

Current affiliation:

Staff Physician

American Pain \& Wellness

Plano, TX 75093

E-mail:mhu@painandwellness.com

Clifford J. Eskey, MD, PhD

Clinical Fellow in Interventional

Neuroradiology

Endovascular Neurosurgery and

Interventional Neuroradiology

Neurosurgical Service and Department

of Radiology

Massachusetts General Hospital

Harvard Medical School

Boston, MA 02114

Samuel C. Tong, MD

Staff Physician

Endovascular Neurosurgery and

Interventional Neuroradiology

Neurosurgical Service and Department

of Radiology

Massachusetts General Hospital

Harvard Medical School

Boston, MA 02114
Raul G. Nogueira, MD

Clinical Fellow in Interventional

Neuroradiology

Endovascular Neurosurgery and

Interventional Neuroradiology

Neurosurgical Service and Department

of Radiology

Massachusetts General Hospital

Harvard Medical School

Boston, MA 02114

Stuart R. Pomerantz, MD

Staff Physician

Endovascular Neurosurgery and

Interventional Neuroradiology

Neurosurgical Service and Department of Radiology

Massachusetts General Hospital

Harvard Medical School

Boston, MA 02114

James D. Rabinov, MD

Staff Physician

Endovascular Neurosurgery and

Interventional Neuroradiology

Neurosurgical Service and Department

of Radiology

Massachusetts General Hospital

Harvard Medical School

Boston, MA 02114
Johnny C. Pryor, MD

Staff Physician

Endovascular Neurosurgery and Interventional Neuroradiology Neurosurgical Service and Department of Radiology

Massachusetts General Hospital

Harvard Medical School

Boston, MA 02114

Joshua A. Hirsch, MD

Radiology section editor, Pain

Physician journal

Director, Endovascular Neurosurgery

and Interventional Neuroradiology

Chief, Minimally Invasive Spine Surgery

Neurosurgical Service and Department of Radiology

Massachusetts General Hospital

Harvard Medical School

Boston, MA 02114

E-mail: jahirsch@partners.org 


\section{References}

1. Tong SC, Eskey CJ, Pomerantz SR, Nogueira RG, Yoo A, Growney M, Rabinov JD, Pryor JC, Hirsch JA. "Sky"phoplasty: A single institution's initial experience. Manuscript accepted for publication, J Vasc Intervent Radiol.

2. Coumans JV, Reinhardt MK, Lieberman IH. Kyphoplasty for vertebral compression fractures: 1-year clinical outcomes from a prospective study. I Neurosurg 2003; 99: 44-50.

3. Nussbaum DA, Gailloud P, Murphy K. A review of complications associated with vertebroplasty and kyphoplasty as reported to the Food and Drug Administration medical device related web site. J Vasc Interv Radiol 2004; 15:1185-1192.

4. Steinmann J, Tingey CT, Cruz G, Dai Q. Biomechanical comparison of unipedicu- lar versus bipedicular kyphoplasty. Spine 2005; 30:201-205.

5. Hoh BL, Rabinov JD, Pryor JC, Hirsch JA. Balloon kyphoplasty for vertebral com pression fracture using a unilateral balloon tamp via a uni-pedicular approach: technical note. Pain Physician 2004; 7:111 114 . 